\title{
Effet du milieu et de la provenance sur le rendement en fibres chez le pin sylvestre (Pinus sylvestris L.) en Finlande
}

\author{
G. NEPVEU et Pirkko VELLING * \\ INRA, Station de Recherches sur la Qualité des Bois \\ Centre de Recherches forestières, Champenoux, F 54280 Seichamps \\ * Institut National de Recherches forestières de Finlande \\ Unioninkatu $40 \mathrm{~A}, \mathrm{SF} 00170 \mathrm{Helsinki}$ (Finlande)
}

\section{Résumé}

La variabilité du rendement en fibres et de l'anisotropie de l'infradensité a été étudiée sur 432 pins sylvestres (19 ans depuis la graine) en utilisant des échantillons non destructifs (carottes de sondage). Six milieux (Côte Sud et Archipel, Centre, Centre Nord-Ouest de la Finlande) et 12 provenances étaient représentés.

Pour les deux caractères étudiés, la variabilité entre provenances n'est pas significative. En revanche, il existc un effet du milieu très net, surtout pour le rendement en fibres $(39,8$ p. 100 pour la Finlande du Centre-Nord-Ouest; 43,5 p. 100 pour la Côte Sud et l'Archipel).

Cette variabilité due au milieu, qui semble suivre un gradient nord-sud, peut jouer un rôle non négligeable sur la qualité du bois de pin sylvestre actuellement utilisé dans les usines de pâtes à papier.

La variabilité due au milieu pour le rendement en fibres n'est expliquéc que partiellement par la production de bois de compression.

Il est suggéré que le climat ou la photopériode pourraient avoir une influence sur les processus de lignification.

Mots clés : Pinus sylvestris, Finlande, provenances, milieux, carottes de sondage, rendement en fibres, bois de compression.

\section{Introduction}

Avec 45 p. 100 du volume de bois sur pied, le pin sylvestre est l'essence principale en Finlande. Il dépasse l'épicéa commun (37 p. 100 du volume sur pied) et le bouleau (15 p. 100) (Metsätilastollinen Vuosikirja, Yearbook of Forest Statistics, 1983).

Ce chiffre explique en partie l'effort que les généticiens forestiers finlandais accordent à cette essence. Ainsi, début 1985 , le pin sylvestre représentait-il 39 p. 100 des arbres sélectionnés enregistrés, 54 p. 100 des clones en collection, 73 p. 100 de la surface des peuplements classés et 89 p. 100 de celle des vergers à graines 
(Suomen Metsänjalostuksen Yleistilastoa 1-1-1985, General Statistics on Forest Tree Breeding in Finland 1-1-1985).

Le bois de pin sylvestre de faibles dimensions, notamment celui provenant de coupes d'éclaircie, étant utilisé dans l'industrie de la pâte à papier, il nous a semblé important dans cet article de nous intéresser au rendement en fibres de ce bois. Ce rendement est défini ici comme le pourcentage en poids anhydre de fibres papetières que l'on peut extraire par cuisson chimique de type Kraft d'une masse de bois anhydre.

Notre but était de répondre à deux questions :

- existe-t-il une variabilité significative entre provenances pour le rendement en fibres chez le pin sylvestre? Une réponse positive à cette question pourrait conduire les généticiens finlandais à inclure cette caractéristique dans les critères de sélection pris en compte dans les programmes d’amélioration de cette essence ;

- existe-t-il un effet du milieu significatif pour le rendement en fibres? La réponse intéresse cette fois les forestiers et les producteurs de pâtes papetières pour lesquels il n'est pas indifférent de savoir si un même poids (anhydre) de bois acheté dans le nord ou le sud de la Finlande est susceptible de produire ou non le même poids de fibres.

Outre ces deux questions, nous aborderons également celle de laanisotropie de la densité du bois qui, si elle est prononcée, peut conduire à des problèmes technologiques assez importants du fait de la production de bois de compression. Cet autre aspect de la qualité du bois chez le pin sylvestre sera évoqué, d'une part sous l'angle de la connaissance de sa variabilité (génétique et due au milieu), d'autre part, en tant que critère explicatif de la variabilité du rendement en fibres.

Nous précisons que cet article est partiellement repris d'un texte plus volumineux signé Pirkko Velling \& G. Nepveu qui sera publié dans la revue Folia Forestalia sous le titre (traduit en français) : "Variabilité de la productivité et de la qualité du bois dans un test multistationnel de provenances de pin sylvestre (Pinus sylvestris) d'origine finlandaise ». Nous remercions M. Aarne Nyyssönen, Directeur de l'Institut National de Recherches Forestières de Finlande, qui nous a autorisés à faire état des résultats que nous présentons aujourd'hui.

\section{Matériel et méthodes}

Les mesures ont porté sur 432 carottes de sondage diamétrales (de $5 \mathrm{~mm}$ de diamètre) orientées nord-sud et prélevées à $1,30 \mathrm{~m}$.

Les 432 carottes de sondage provenaient de 432 arbres répartis de manière parfaitement équilibrée en 12 provenances répétées dans 6 dispositifs expérimentaux (fig. 1). Ces dispositifs avaient été installés en 1966 par Max. Hagman, aujourd'hui responsable du Département de Génétique Forestière de l'Institut National de Recher. ches Forestières de Finlande, dans le cadre d'une expérimentation très vaste comportant 17 à 30 provenances dans 11 sites expérimentaux, expérimentation dont nous avons extrait notre sous-échantillonnage par souci d'équilibre statistique. Chacun des dispositifs étudiés ici était constitué de 6 blocs complets avec des parcelles unitaires de 25 plants distants de $2 \mathrm{~m}$ en tous sens; dans chacune de ces parcelles, 1 arbre pris au hasard a été sondé. 


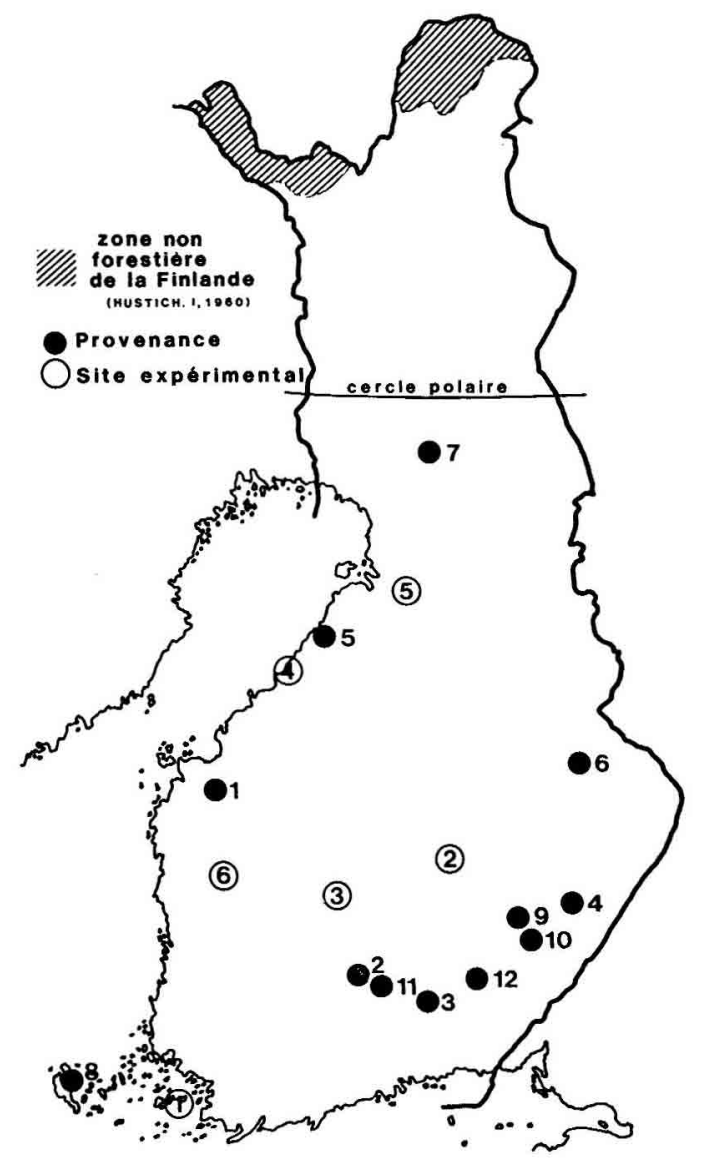

FIG. 1

Localisation des provenances et des sites expérimentaux étudiés.

Location of provenances and experimental sites studied.

Au moment du sondage, les arbres étaient âgés de 19 ans depuis la graine. Sur chaque carotte de sondage, on a mesuré :

- les infradensités du bois des rayons nord et sud de la carotte. Ces infradensités ont été obtenues par la méthode de saturation intégrale (KEYLWERTH, 1954) après enlèvement de la résine par passage dans un soxhlet. On a ensuite calculé une anisotropie de l'infradensité du bois de la manière suivante :

$$
\text { anisotropie de l'infradensité du bois }=\frac{\text { infradensité du rayon à forte infradensité }}{\text { infradensité du rayon à faible infradensité }}
$$


On l'appellera par la suite anisotropie de la densité du bois (à noter que cette formule est différente de celle adoptée dans l'article de base signalé plus haut, article de base dans lequel nous nous intéresserons à l'anisotropie systématique nord-sud);

- le rendement en fibres $\left(\frac{\text { poids de fibres }}{\text { poids de bois }} \times 100\right)$ pour l'ensemble de la carotte de sondage en utilisant la méthodologie décrite par JaNIN (1972 a et 1983). Les conditions de cuisson étaient les suivantes : cuisson de type Kraft, taux d'alcali actif : 22 p. 100 , sulfidité : 25 p. 100, rapport liquide/bois : 4. L'effet «cuisson» avait été corrigé.

\section{Résultats et discussion}

\subsection{Existence d'un gradient nord-sud pour le rendement en fibres et l'anisotropie de la densité da bois}

Le tableau 1 présente les résultats de l'analyse de variance réalisée pour le rendement en fibres et l'anisotropie de la densité du bois.

\section{TABlenu 1}

Effets du milieu et de la provenance sur le rendement en fibres et l'anisotropie de la densité du bois chez le pin sylvestre.

Résultats des analyses de variance.

Environmental and genetic effect on fiber yield and anisotropy of wood density in Pinus sylvestris. Results of variance analysis.

\begin{tabular}{|c|c|c|c|}
\hline \multirow[b]{2}{*}{ Sources de variation } & \multirow[b]{2}{*}{$\begin{array}{l}\text { Degrés de } \\
\text { liberté } \\
\text { Degrees of } \\
\text { freedom }\end{array}$} & \multicolumn{2}{|c|}{ F calculés } \\
\hline & & $\begin{array}{c}\text { Rendement en fibres } \\
\text { Fiber yield }\end{array}$ & $\begin{array}{c}\text { Anisotropic } \\
\text { de la densité du bois } \\
\text { Anisotropy } \\
\text { of wood density }\end{array}$ \\
\hline $\begin{array}{l}\text { Provenance } \ldots \ldots \ldots \ldots \ldots \ldots \\
\text { Provenance }\end{array}$ & 11 & $1,43 \mathrm{NS}$ & $<1 \mathrm{NS}$ \\
\hline $\begin{array}{l}\text { Milieu } \\
\text { Site }\end{array}$ & 5 & $45,06^{* *}$ & $5,83^{* *}$ \\
\hline $\begin{array}{l}\text { Interaction provenance } \times \text { milieu } \\
\text { Provenance } \times \text { site interaction }\end{array}$ & 55 & $<1 \mathrm{NS}$ & $<1 \mathrm{NS}$ \\
\hline $\begin{array}{l}\text { Bloc dans milieu } \ldots \ldots \ldots \ldots \ldots \\
\text { Block in site }\end{array}$ & 30 & $1,13 \mathrm{NS}$ & $1,24 \mathrm{NS}$ \\
\hline $\begin{array}{l}\text { Variabilité résiduelle } \ldots . . \\
\text { Residual variability }\end{array}$ & 330 & - & 一 \\
\hline Total $\ldots \ldots \ldots \ldots \ldots \ldots \ldots$ & 431 & & \\
\hline
\end{tabular}

NS : Effet non significatif - Not significant effect.

**: Effet significatif au seuil de 1 p. 100 - Effect significant at I p. 100 level. 
Les enseignements très clairs de ce tableau sont sensiblement les mêmes pour les deux caractères : l'effet «provenance» n'est pas significatif alors que l'effet du milieu apparaît très net, notamment pour le rendement en fibres avec une valeur de $F$ à 5 et 330 degrés de liberté de 45,06 !

Comme l'indique le tableatu 2, les différences entre dispositifs ne sont pas négligeables; malgré le faible nombre de dispositifs étudiés (6), les résultats suggèrent l'existence d'un gradient nord-sud assez net pour les deux caractères : globalement, le rendement en fibres diminue et l'anisotropie de la densité du bois augmente (donc la qualité du bois diminue) au fur et à mesure que l'on progresse vers le nord. Pour le rendement en fibres, notre observation rejoint très bien les conclusions formulées par KALLA (1966) à la suite de mesures réalisées dans des laboratoires d'entreprises finlandais; en ce qui concerne l'anisotropie de la densité du bois, nous ne sommes pas très surpris de ce résultat car nous savons que, dans des conditions de milieu relativement difficiles, les conifères qui présentent alors une forme assez mauvaise réagissent en produisant une proportion notable de bois de compression (POLGE \& ILLY, 1967) : c'est le cas des dispositifs 4 et 5 dans lesquels les arbres présentent d'ailleurs une forme médiocre attribuée dans le premier cas essentiellement au vent et à la nature marécageuse du sol, dans le second à des dégâts de rouille (Melampsora pinitorqua).

TABLEaU 2

Rendement en fibres et anisotropie de la densité du bois en fonction de la position géographique pour le pin sylvestre en Finlande.

Fiber yield and anisotropy of wood density in relation to geographical location for Pinus sylvestris in Finland.

\begin{tabular}{|c|c|c|c|}
\hline \multicolumn{2}{|c|}{$\begin{array}{l}\text { Région géographique } \\
\text { Geographical area }\end{array}$} & $\begin{array}{l}\text { Rendement } \\
\text { en fibres }(\%) \\
\text { Fiber yield }\end{array}$ & $\begin{array}{l}\text { Anisotropie de } \\
\text { la densité du bois } \\
\text { Anisotropy } \\
\text { of wood density }\end{array}$ \\
\hline $\begin{array}{c}\text { Finlande du } \\
\text { Centre-Nord-Ouest } \\
\text { Central-Northwestern } \\
\text { Finland }\end{array}$ & $\begin{array}{l}\text { Dispositif } 5 \quad \ldots \ldots \\
\text { Experimental site } \\
\text { Dispositif } 4 \quad \ldots \ldots\end{array}$ & $\begin{array}{l}39,7 \\
39,8\end{array}$ & $\begin{array}{l}1,08 \\
1,08\end{array}$ \\
\hline $\begin{array}{l}\text { Finlande } \\
\text { du Centre } \\
\text { Central } \\
\text { Finland }\end{array}$ & $\begin{array}{l}\text { Est : Dispositif } 2 \\
\text { Centre : Dispositif } 3 \\
\text { Ouest : Dispositif } 6\end{array}$ & $\begin{array}{l}40,7 \\
41,9 \\
41,6\end{array}$ & $\begin{array}{l}1,04 \\
1,04 \\
1,04\end{array}$ \\
\hline $\begin{array}{c}\text { Finlande du Sud } \\
\text { (Côte et Archipel) } \\
\text { Southern part of Finland } \\
\text { (Coast and Archipelago) }\end{array}$ & Dispositif 1 & 43,5 & 1,03 \\
\hline
\end{tabular}

Le génotype moyen est le même dans les 6 milieux considćrés Mean genotype is the same in the 6 experimental sites studied. 
Nous insisterons sur le fait que ces résultats correspondent bien à un effet purement environnemental puisque la composition génétique était strictement la même dans les 6 sites considérés (les mêmes provenances y sont représentées). Pour être complet, notons que le dispositif situé dans le Centre Est (dispositif 2) présente un rendement en fibres un peu plus faible que les dispositifs du centre de la Finlande situés plus à l'Ouest (dispositifs 3 et 6 ).

Au gradient principal nord-sud, semble donc s'ajouter un gradient est-ouest pour la Finlande du Centre.

\subsection{Conséquence industrielle de la variabilité nord-sud du rendement en fibres}

Les informations contenues dans les tableaux 1 et 2 ne sont pas entièrement propres à répondre aux préoccupations de l'utilisateur actuel de bois pour la production de fibres papetières. En effet, uelui-ci achète présentement dans le Nord du pays des provenances septentrionales et dans le Sud des provenances méridionales.

C'est pourquoi, sur la base de nos résultats, nous présentons dans le tableau 3 les poids moyens de fibres qu'un tel industriel peut attendre de $100 \mathrm{~kg}$ de bois anhydre en fonction de ses sources d'approvisionnement. Les rendements en fibres de ces sources d'approvisionnement sont représentés par les rendements des provenances et des dispositifs de notre échantillonnage que nous avons considérés représentatifs de la zone du territoire finlandais considérée.

Ces chiffres, à considérer dans ce cas précis avec prudence étant donné la faiblesse de l'échantillonnage des provenances et des milieux, sont très proches de ceux qui figuraient dans le tableau 2. Ceci n'est pas très surprenant étant donné les résultats de l'analyse de variance indiqués au tableau 1 (absence d'effet provenance, absence d'interaction provenance $X$ milieu).

Le producteur de pâte à papier de pin sylvestre qui paierait le bois sur la base de son poids sec pourrait donc avoir intérêt à accorder un meilleur prix aux bois provenant du Sud du pays (ceci sans préjuger d'une éventuelle variabilité due au milieu pour l'aptitude au blanchiment de la pâte en question).

Remarque : Dans l'article «de base » signé Pirkko Vell.ing \& G. NepVeu, nous reprendrons cette question en comparant les gains ou les pertes en fonction de ses sources d'approvisionnement d'un producteur de fibres papetières qui paierait la matière première sur la base de son poids sec ou de son volume. Pour être complet, il aurait été intéressant d'évoquer le cas d'un industriel qui paierait cette matière première sur la base de son poids frais. Nous n’avons malheureusement pas pu traiter cette question dans le cadre de ces deux articles faute de disposer de données concernant l'humidité du bois.

\subsection{Effet de l'anisotropie de la densité du bois sur le rendement en fibres}

Au niveau des moyennes de dispositifs, le tableau 2 suggérait l'existence d'une liaison significative entre le rendement en fibres et l'anisotropie de la densité du bois. 


\section{TABLeaU 3}

Poids moyen de fibres attendu pour 1 tonne de bois anhydre en fonction des sources d'approvisionnement d'une usine de pâte.

Mean weight of fibers expected from 1 ton of dry wood in relation to wood supply areas for a pulp mill.

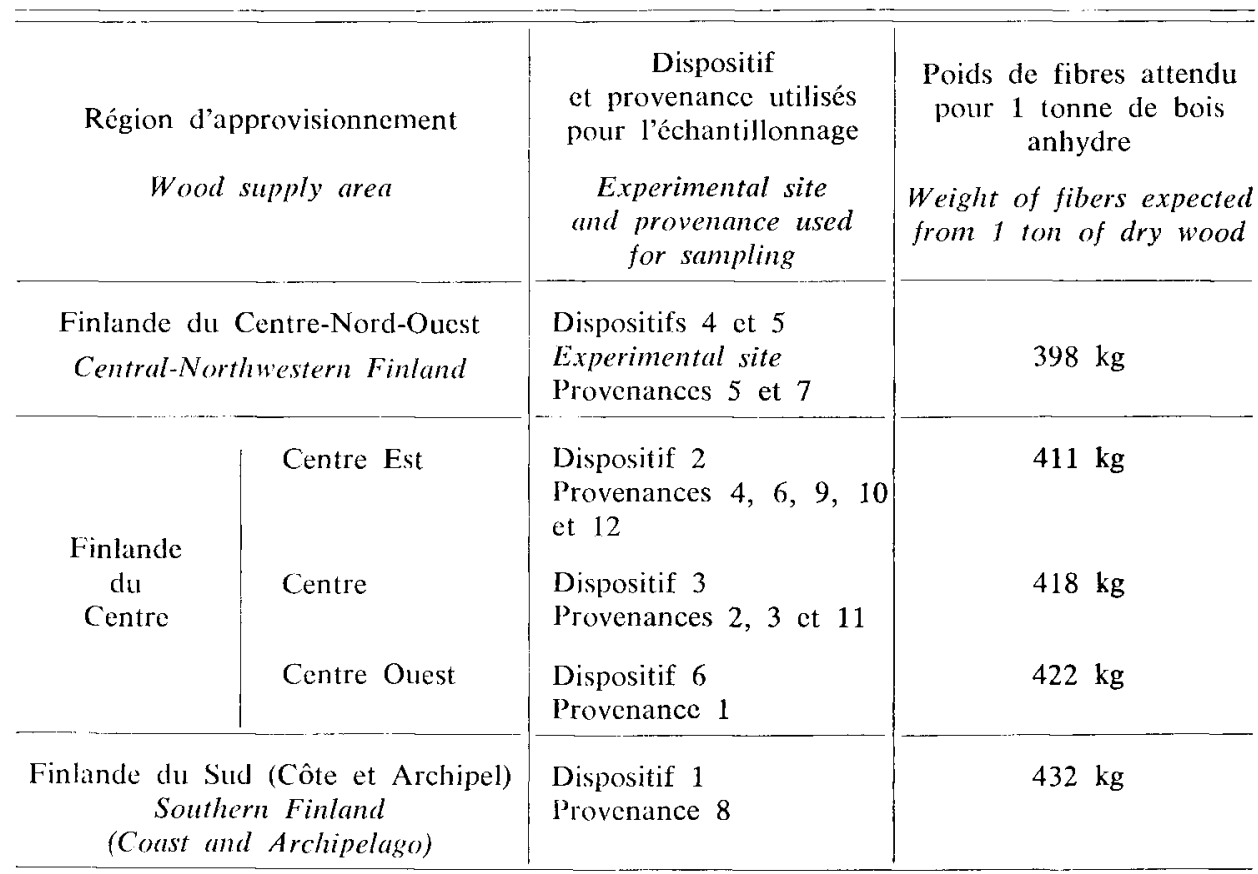

Les rendements en fibres des sources d'approvisionnement sont représentés par les rendements des provenances et des dispositifs de notre échantillonnage que nous avons considérés représentatifs de la zone du territoire finlandais conce! née.

Fiber yields of wood supply areas are represented by fiber yields of provenances or experimental siles in our sampling which are considered to be representative for concerned area of finnish rerritory.

A l'intérieur des dispositifs, cette relation se maintient de manière très nette comme en témoigne la figure 2; celle-ci montre que, lorsque l'anisotropie de la densité augmente, le rendement en fibres diminue de manière significative.

Cette observation rejoint très bien celles faites antérieurement sur d'autres conifères, en particulier sur Pinus pinaster (Polge \& ILly, 1967, op. cit.; JANIN, 1972 b ; Orta et al., 1985) : une forte anisotropie de densité du bois témoigne de la formation d'une quantité importante de bois de compression, lequel est caractérisé par un taux élevé de lignification, responsable d'un rendement en fibres faible. 


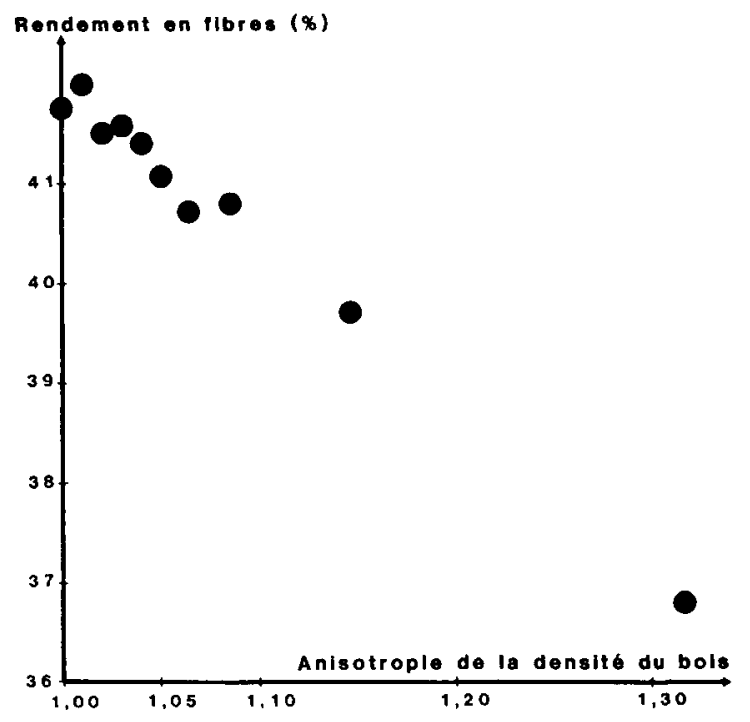

Fig. 2

Effet de l'anisotropie de la densité du bois sur le rendement en fibres.

Effect of anisotropy of wood density on fiber yield in Pinus sylvestris.

Niveau intradispositif. Pour chaque valeur d'anisotropie, le rendement en fibres est la moyenne des moyennes des sites expérimentaux. Chaque point est la moyenne d'au moins 17 observations.

Intrasite level. For each value of anisotropy, fiber yield is the mean of experimental site means. Each point is the mean of at least 17 individual measurements.

2.4. La variabilité nord-sud pour l'anisotropie de la densité du bois explique-t-elle à elle seule le gradient nord-sud observé pour le rendement en fibres?

Pour répondre à cette question, nous avons procédé à deux analyses de variance sur les valeurs de rendements en fibres, la première avec l'ensemble de l'échantillonnage initial (432 individus), la seconde avec les arbres dont l'anisotropie de la densité du bois était au plus égale à 1,05 (315 individus); cette règle nous a permis, tout en conservant un effectif d'arbres confortable, d'exclure de la seconde analyse statistique les individus dont la faiblesse du rendement en fibres résultait principalement d'une formation importante de bois de compression (voir fig. 2).

Dans les deux cas, nous avons procédé à une analyse de variance à un facteur, l'effet contrôlé étant celui du site. Les résultats indiquent que le $F$ dispositif reste très significatif en excluant le bois de compression $\left({ }_{3: 19}^{5} \mathrm{~F}=31,07 * *\right.$ contre ${ }_{4: 6}^{5} \mathrm{~F}=$ $45,90^{* *}$ pour l'ensemble des individus).

La figure 3 illustre ce propos : clle montre que l'anisotropie de la densité du bois - qui témoigne de la formation du bois de compression - n'explique que de manière partielle l'effet très significatif du milieu (gradient nord-sud) pour le rendement en fibres chez le pin sylvestre en Finlande. 


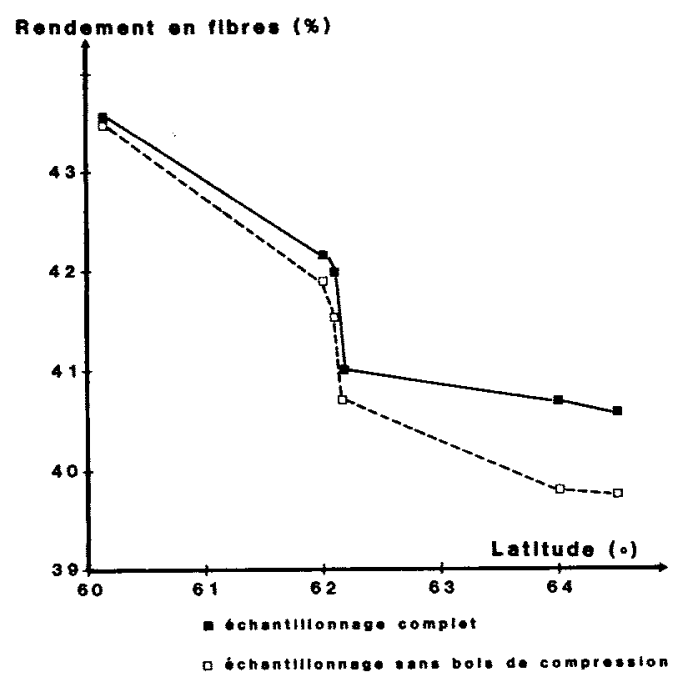

FIG. 3

Effet de la latitude du site expérimental sur le rendement en fibres chez Pinus sylvestris en Finlande.

Effect of the experimental site latitude on fiber yield in Pinus sylvestris in Finland.

Rendement en fibres - Fiber yield.

Latitude du site expérimental - Latitude of experimental site.

Echantillonnage complet - Complete sampling.

Echantillonnage sans bois de compression - Sampling without compression wood.

\section{Conclusions}

Bien que l'échantillonnage - très satisfaisant sur le plan de l'effectif et de sa composition génétique - ait été relativement faible du point de vue du nombre de milieux représentés, les résultats nous apparaissent suffisamment nets pour avancer, d'une part des conclusions d'ordre technologique, et d'autre part, ceci modestement, des hypothèses physiologiques.

\subsection{Conclusions «technologiques»}

Si la variabilité génétique infraspécifique (variabilité entre provenances) apparaît non significative pour le rendement en fibres chez le pin sylvestre (ce qui rejoint assez bien bon nombre d'observations faites sur d'autres conifères), un effet milieu très net, assorti d'une variabilité importante, a été mis en évidence. Cet effet «milieu» semble obéir à un gradient nord-sud, les bois provenant du Sud du pays présentant le rendement en fibres le plus élevé.

Un autre caractère qualitatif important, l'anisotropie de la densité du bois, semble suivre le même type de variabilité, avec toutefois un gradient nord-sud un peu moins net. 


\subsection{Conclusions "physiologiques》}

Le gradient nord-sud mis en évidence pour le rendement en fibres n'est que partiellement expliqué par la variabilité entre sites significative pour l'anisotropie de la densité du bois.

Ceci suggère que le climat, la durée du jour, qui varient considérablement en fonction de la latitude en Finlande au cours de la saison de végétation, pourraie.st être responsables de processus de lignification différents suivant les sites considérés ou, à tout le moins, d'équilibres lignine - hémicellulose - cellulose variables.

Cette hypothèse, qui avait déjà été formulée par Kalla (1966, op. cit.), mériterait d'être vérifiée sur un échantillonnage de sites plus représentatifs (en incluant par exemple des milieux plus méridionaux que nous le permet le territoire finlandais) ou, à défaut, en élevant des plants de génotypes bien identifiés en conditions contrôlées.

$$
\begin{aligned}
\text { Reçu en juin } 1985 . \\
\text { Accepté en septembre } 1985 .
\end{aligned}
$$

\section{Summary \\ Environmental and genetic effects on fiber yield in Scots pine (Pinus sylvestris) in Finland}

Variability for fiber yield and anisotropy of wood density were studied in 432 Pinus sylvestris (19 years from the seed) by using non-destructive samples (increment cores). Six environments (Southern coast and archipelago of Finland, central Finland, CentralNorthwestern Finland) and twelve provenances were sampled (fig. 1).

For the two characteristics studied, variability between provenances is not significant. On the other hand, there is a highly significant environmental effect, particularly for fiber yield : from 39.8 p. 100 in Central-Northwestern Finland to 43,5 p. 100 in Southern coast and archipelago of Finland (tabl. 1, tabl. 2).

This environmental variability which seems to follow a North-South gradient can have a significant effect on wood quality of Pinus sylvestris used at present for pulping (tabl. 3).

The formation of compression wood is only one of the factors accounting for environmental variability of fiber yield (fig. 2, fig. 3 ). process.

The authors suggest that climate or photoperiod could have an effect on lignification

Key words : Pinus sylvestris, Finland, provenances, environments, increment cores, fiber yield, compression wood.

\section{Zusammenfassung}

Einfluss der Umwelt und der Provenienz auf die Zellstoffausbeute der gemeinen Kiefer (Pinus sylvestris) in Finnland

Die Variabilität von Zellstoffausbeute und Anisotropie der Holzdichte wurde an 432 gemeinen Kiefern untersucht (19 Jahre seit der Saat) mit Hilfe von nicht-zerstörenden Proben (Bohrkerne). Sechs Umwelttypen der finnischen Gebiete (südliche Küste und Archipel, Zentrum, Zentrum-Nord-West) und 12 Provenienzen waren vertreten. 
Für die zwei untersuchten Merkmale ist die genetische Variabilität nicht signifikant. Es gibt hingegen ein schr bedeutendes Süd-Nordgefälle vor allem für die Zellstoffausbeute (43,5 p. 100 für der südlichen Küste und dem Archipel ; 39,8 p. 100 für Zentrum-Nord-West).

Diese Nord-Südvariabilität kann einen nicht unbedeutenden Einfluss auf die gegenwärtig in den Zellstoffindustrien gebrauchte Holzqualität der gemeinen Kiefer ausüben.

Die der Umwelt unterworfene Variabilität der Zellstoffausbeute kann nur teilweise durch die Produktion von Druckholz erklärt werden. könnten.

Es wird nahegelegt, dass Klima und Photoperiode den Verholzungsprozess beeinflussen

Schlïsselwörter : Pinus sylvestris, Finnland, Provenienzen, Umwelt, Bohrkerne, Zellstoffausbeute, Druckholz.

\section{Références bibliographiques}

Hustich I., 1960. Plant geographical regions. A Geography of Norden, Ed. A. Semme, 364 p., Oslo-Bergen, 54-62.

JANIN G., 1972 a. Microcuissons papetières. Méthode adaptée aux recherches forestières portant sur la détermination des caractéristiques papetières individuelles sur arbres vivants à l'aide d'échantillons de bois dont le mode de prélèvement, l'aspect et le poids ne sont pas usuels. La Papeterie, $\mathrm{n}^{\circ} 3,13 \mathrm{p}$.

JANIN G., 1972 b. Le diagramme polaire différenticl des rendements, nouveau critère d'homogénéité des bois au point de vue des propriétés papetières. Document à distribution limitée, Station de Recherches sur la Qualité des Bois, C.N.R.F., nº 1972/8, 16 p.

JANIN G., 1983. Microtests papetiers. Microcuisson, microclassage, microraffinage, mesure automatique de la longueur des fibres. Thèse de Docteur d'Etat es-Sciences, INPG, 5 mai, 235 p. + annexes.

Keylwerth R., 1954. Ein Beitrag zur qualitativen Zuwachsanalyse. Holz als Roh und Werkstoff, 12, 77-83.

KALla J., 1966. (Mäntypinotavaran kulutus valkaisemattoman sulfaattisclluloosan valmistuksessa pohjois - ja ctelä-suomessa). Summary : The consumption of pine pulpwood in the preparation of unbleached sulphate cellulose in northern and southern Finland. Metsï̈tuloudellinen aikakauslehti, 83, 149-160.

METSÄTILASTOLLINEN VUOSIKIRJA. Skogsstatistisk ärsbok. Yearbook of forest statistics, 1983. Suomen Virallinen Tilasto. Finlands Officiclla statistik. Official Statistics of Finland. XVII A : 13. Folia Forestalia, 590.

Ohta S., Keller R., Janin G., 1985. Effets de divers modes de fertilisation (N, P, K) sur certaines caractéristiques physiques, chimiques, mécaniques et propriétés papetières du pin maritime des Landes (Pinus pinaster Ait.). II. Bois de compression et propriétés papetières. Ann. Sci. For., 42 (1), 69-96.

Polge H., Illy G., 1967. Observations sur l'anisotropie du pin maritime des Landes. Ann. Sci. For., 24 (3), 205-231.

SUOMEN METSÄNJALOSTUKSEN YLEISTILASTOA 1-1-1985, General statistics on forest tree breeding in Finland 1-1-1985. The Finnish Forest Research Institute, Department of Forest Genetics, National register, 20 p. 